\title{
ROLE OF PSYCHOLOGICAL EFFECTS OF REAL-TIME TRAVEL INFORMATION PROVISION ON EN ROUTE TRAVELER ROUTE CHOICE DECISIONS
}

\author{
Dongyoon Song ${ }^{1}$, Srinivas Peeta ${ }^{1}$, Yu-ting $\mathrm{Hsu}^{2}$, Shubham Agrawal ${ }^{1}$ \\ ${ }^{1}$ Purdue University, IN, USA, ${ }^{2}$ National Taiwan University, Taiwan \\ Email: peeta@purdue.edu
}

\begin{abstract}
Summary: While real-time travel information can aid travelers to make informed decisions, it may increase the cognitive load in information perception and the complexity in decision-making process, especially when the information is from multiple sources. Under this circumstance, human factors-related aspects in information perception and the consequent psychological effects of the information play significant roles in traveler route choice decision-making. This study proposes a hybrid route choice model under the presence of real-time travel information, in which latent variables are employed to represent the psychological effects of information provision. Three dimensions of psychological effects - cognitive burden, cognitive decisiveness, and emotional relief - are assumed as latent psychological constructs in the proposed model. Data on traveler behavior and information perception are obtained through interactive driving simulator-based experiments. Estimation results will verify that the inclusion of the latent variables enhances the understanding of route choice decision-making under information provision.
\end{abstract}

\section{INTRODUCTION}

Recent advances in information and mobile communications technologies associated with Advanced Traveler Information Systems (ATIS) have led to the increased use of smart devices (such as smartphones or in-vehicle online console panels) while driving, and altered travelers' patterns of real-time travel information use. The ability to receive information through multiple delivery formats (such as text, voice, and image) and multiple sources at different times and locations, in conjunction with the inherent need to multitask due to the interactive nature of the travel environment, raises fundamental questions related to the psychological effects of information provision. That is, what are the relationships involving drivers' cognitive capabilities, information content and characteristics, and information delivery mechanisms? How do these relationships impact the effectiveness of the provided information and user satisfaction with it? As user information systems mature, and enter the realm of widespread deployment, addressing these questions is critical to ensuring that travelers make informed travel decisions while having satisfactory travel experiences, and for the effective design of ATIS and information delivery. They have implications for the safety and reliability of information-based travel systems, policy-maker justifications of ATIS-related investments, and information delivery strategies for information service providers.

Traveler route choice behavior under information provision has been generally acknowledged to be affected by traveler attributes, situational factors, route characteristics, and the provided information to different extents (Jha et al., 1998; Avineri and Prashker, 2006; Ben-Elia and Shiftan, 2008). Especially, information content has been shown to elicit differential behavioral 
responses. For instance, the effects of information have been studied for its ability to persuade travelers to shift routes (Peeta et al., 2000) and information-related behavioral phenomena associated with the day-to-day and within-day effects of information on route choice behavior (Srinivasan and Mahmassani, 2000; Nakayama and Kitamura, 2000; Peeta and Yu, 2004; 2005; $\mathrm{Yu}$ and Peeta, 2011). In terms of qualitative aspects of information perception, Bonsall (2004) and Chorus et al. (2006) show that traveler route choice decisions rely on the subjective perception of the provided information associated with traveler attributes and situational factors. That is, even if the same information is provided to travelers under similar traffic conditions, their route choice decisions may differ because the information is perceived and used differently by different travelers. Following this thread, different approaches have been used to study the effects of real-time travel information by factoring the qualitative aspects of information perception. For instance, well-defined behavioral theories on the limitations or distortions in human cognition and reasoning, such as bounded rationality (Gao et al., 2011) or prospect theory (Razo and Gao, 2013), are leveraged to develop modeling structures that account for the qualitative aspects of travelers' behavioral responses to real-time travel information. However, an underlying assumption in these studies is that travelers can seamlessly process information that they receive in a driving environment; that is, human factors such as cognitive load are not factored in the response. More importantly, no study has explicitly addressed the role of information perception in decision-making process which can possibly lead to challenges such as information overload and information-induced confusion. These perception aspects need to be holistically considered in route choice decision-making process to enable realism in inferring driver behavior under information provision in the inherently interactive multitasking driving environment. In this study, we propose the concept of an information-related psychological process to explicitly illustrate the role of information perception and its implications for route choice decision-making using latent psychological constructs.

In this study, four facets of information perception - ease of comprehension, sufficiency, consistency, and favorableness - are specified to characterize the psychological effects of information provision. Ease of comprehension implies information perception in terms of cognitive complexity (how clearly the information is presented) and cognitive load (amount of information) of the provided information. Sufficiency implies information perception in terms of whether the provided information satisfies traveler's information needs for informed decisionmaking. Consistency represents information perception in terms of the consistency between: (i) the provided information and past travel experience, or (ii) information from multiple sources. Favorableness refers to information perception in terms of whether the provided information is favorable to the traveler's trip context (for example, based on the trip purpose or destination); that is, travel conditions implied in the provided information are desirable for the specific trip being made. Based on the information perception and other explanatory factors (such as traveler attributes and situational factors), three psychological effects of real-time travel information cognitive burden, cognitive decisiveness, and emotional relief - are assumed to affect route choice decision-making. Cognitive burden refers to the amount of mental effort that needs to be expended in processing information-related cues in the driving environment. Cognitive decisiveness refers to the level of awareness in comprehending the travel situation, and the level of uncertainty reduction in making decisions, based on the provided information. Emotional 
relief refers to the level of mental relief due to the anticipation of future outcomes, based on the provided information. Figure 1 illustrates the roles of the latent psychological effects (dashed lines and arrows) in the conventional structure of route choice decision-making process under information provision. These latent psychological constructs will be identified through observed indicators collected by driving simulator experiments.

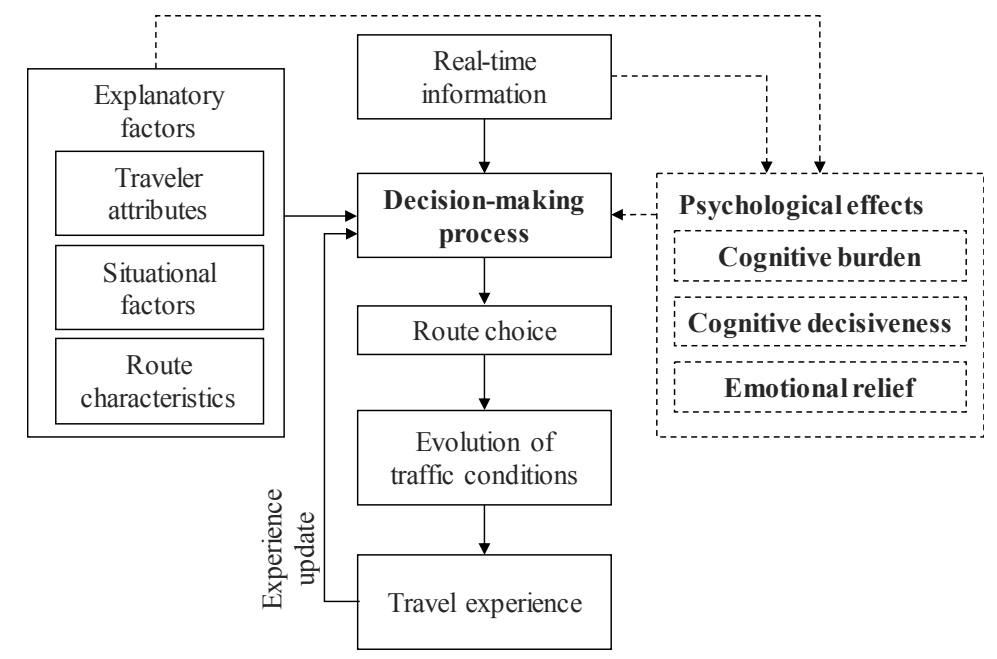

\section{METHODS}

Figure 1. Route choice decision-making process under real-time travel information provision

\section{Participants}

A total of 206 participants (103 male, 103 female) between the ages of 19 and 69 (Mean=32.7, $\mathrm{SD}=11.63$ ) are recruited from the university and local communities for the experiment. As each participant completed multiple runs with different travel and information scenarios, a total of 722 observations of route choice decision-making under information provision are obtained from the 206 participants and used in the analysis.

\section{Experiment Design and Procedure}

OKTAL's SCANeR Studio ${ }^{\circledR} 1.4$ is used to create terrain, and satellite images provided by Google Maps are used to construct the network of urban freeways and major arterial roads in northern Indianapolis, Indiana. A single origin-destination (O-D) pair with two route alternatives (freeway and arterial) is selected for the experiment. Each alternative route has a potential location for real-time travel information provision followed by an opportunity to switch the route during the trip. The driving simulator is integrated with a microscopic traffic simulator (AIMSUN), and the traffic conditions created in the microscopic traffic simulator are populated in real-time in the driving simulator. Bi-directional interactions between the two simulators enable traffic events created in the driving simulator (such as accidents) to impact the evolution of microscopic traffic flow in the traffic simulator, so that the information of event occurrence is consistent with the ambient traffic conditions. To avoid playing-a-game attitude and enhance the realism in driving and routing behavior of participants, a point-based reward-penalty system is adopted in the experiments. The points are deducted per a participant's irrational or reckless driving detection (such as excessive speeding and collision) as well as late arrival which also creates motivation to choose the route. The final monetary compensation for a participant is determined based on the points at the end of the experiment. The traffic demand level, familiarity with the alternative route, and trip purpose are controlled in the experiments. The integrated microscopic traffic simulator generates different traffic conditions consistent to the information to provide. Participants' different familiarity level is manipulated by a GPS device that illustrates the alternative route. Two trip purposes are considered: business and non-business. To replicate different levels of sensitivity to travel time and urgency, participants assigned to a business trip are asked to arrive at the destination within a shorter time limit. Incorporating with the point 
system, this travel time restriction can create a different level of incentive to switch the route depending on trip purpose.

In terms of information scenario, one of three options is provided: (i) expected travel time on current route, (ii) expected travel time on current and alternative routes, (iii) expected travel time on current and alternative routes, and prescriptive information (route recommendation). The information is disseminated through one and/or two information sources as follows: (i) variable message sign-based message, if participant currently uses the freeway route, (ii) voice message through a smartphone, and (iii) both these sources if freeway route is currently used. While the VMS message consists of the expected travel time on the freeway route to the next few exits, smartphone-based voice message includes the expected travel time to the destination on the current route or both the current and alternative routes.

The experiments consist of three stages: (i) pre-experiment preliminary survey, (ii) practice session on the driving simulator, and (iii) main session involving multiple driving simulator runs and within-run surveys. In the preliminary survey, demographic and socioeconomic attributes of participants are obtained. Through the practice session, participants are familiarized with driving in the simulator environment. One attribute considered in the study is familiarity with an alternative route. The practice session is designed to construct two levels of familiarity (familiar and unfamiliar groups) with the alternative route using different practice scenarios for each group. In the main session, between three and five runs are presented to participants using various travel scenarios in a random order. The participants choose their routes to the destination, and drive in real-time on it for each scenario with real-time travel information provided based on the scenario. At the point information is provided, a survey is conducted immediately after the potential route switch location to avoid lagged memory bias (that is, to ensure that route decisions are revealed immediately after a switch location if information is provided upstream), and data is collected on the indicator variables for that participant's latent psychological effects. Each within-run survey consists of seven statements $\left(Z^{1}\right.$ to $Z^{7}$ in Table 2$)$ that the participants are asked to respond to using a 7-point Likert scale of 1 to 7 , where 7 indicates strong agreement with the statement.

\section{Model Specification}

A hybrid choice modeling framework (Walker and Ben-Akiva, 2002) based on the multiple indicators multiple causes (MIMIC) structure (Bollen, 2002) is adopted to investigate route choice decision-making with the consideration of psychological effects of information provision. The proposed framework consists of components: (i) a latent variable model and (ii) a hybrid choice model for route choice decision-making. In the latent variable model, the psychological constructs are inferred based on information perception indicator variables (Equation 1), while the impacts of other explanatory variables (such as traveler attributes, situational factors, route characteristics and information characteristics) on the psychological constructs are considered (Equation 2). On the other hand, the utility function of the proposed hybrid route choice model includes both the latent variables for psychological effects and observed explanatory variables (Equations 3 and 4). Here, the revealed route choice behavior, the dependent variable, has a binary choice: staying on the current route or switching to the alternative one. 


$$
\begin{aligned}
& Z_{n}=\mu^{Z} P_{n}+\delta_{n}^{Z} \quad \delta_{n}^{Z} \sim N\left(0, \Sigma_{\delta_{n}^{Z}}\right) \\
& P_{n}=\gamma^{P} X_{n}+\zeta_{n}^{P} \quad \zeta_{n}^{P} \sim N\left(0, \Sigma_{\zeta_{n}^{P}}\right) \\
& U_{\text {in }}=\beta_{X} X_{n}+\beta_{P} P_{n}+\varepsilon_{\text {in }} \quad \varepsilon_{\text {in }} \sim \operatorname{Gumbel}\left(0, \Sigma_{\varepsilon_{\text {in }}}\right) \\
& Y_{\text {in }}=\left\{\begin{array}{cc}
1 & \text { if } U_{\text {in }}=\max _{j}\left\{U_{j n}\right\} \\
0 & \text { otherwise }
\end{array}\right.
\end{aligned}
$$

where, $Z_{n}$ is a vector of indicator variables for individual $n, P_{n}$ is a vector of latent variables for individual $n$, and $\mu^{Z}$ is a matrix of coefficients indicating factor loadings. $\gamma^{P}$ is the coefficient vector for the other (observed) explanatory variables $X_{n}$ which include traveler attributes, situational factors, route characteristics and information characteristics. The measurement error $\delta_{n}^{Z}$ and the structural error $\zeta_{n}^{P}$ are assumed to be independently and identically multivariate normally distributed. $U_{i n}$ is the random utility of alternative $i$ for individual traveler $n, P_{n}$ is a vector of latent variables for traveler $n$ identified in the latent variable model, and $\beta_{P}$ is the coefficient vector of $P_{n} . \beta_{X}$ is the coefficient vector for the other explanatory variables $X_{n}$. The disturbance term $\varepsilon_{i n}$ is independently and identically Gumbel distributed.

\section{ESTIMATION RESULTS}

Table 1 presents the results of the latent variable model with the anchor variables set to 1 to scale the effects of multiple indicators for each latent variable. The key findings related to information characteristics and psychological constructs are following. While higher amount of information expectedly increases cognitive burden, it helps travelers to have an improved cognitive decisiveness regarding the traffic situation. Cognitive decisiveness is also enhanced by alternative route information provision. In contrast, emotional relief is particularly influenced by GPS navigation information of alternative route rather than real-time travel information.

The role of these psychological effects of information provision on the route choice decisionmaking are statistically significant (Table 2). The negative sign for cognitive burden implies that if all other conditions remain equal, travelers with higher cognitive load are more likely to stay on the current route. This implies that the stress from information overload or informationinduced confusion can weaken the influence or effectiveness of information to alter travelers' route choice. By contrast, cognitive decisiveness has a positive sign, which is consistent with the reduced uncertainty and the consequent willingness to take risk in route choice decision-making. If travelers have more clarity on the ambient traffic conditions on the alternative route (higher cognitive decisiveness), they are more likely to choose it when it has better traffic conditions. Emotional relief also has a positive impact on switching route to the alternative. That is, if a traveler feels the information is favorable (for example, available option of alternative route with a shorter expected travel time), he/she would change to the route.

\section{CONCLUDING COMMENTS}

Incorporating the psychological effects of real-time travel information provision can improve the understanding of travelers' route choice decision-making behavior. Comprehensive experiments are designed using an interactive driving simulator integrated in real-time with a microscopic traffic simulator. The estimation results illustrate that the proposed hybrid route choice model, through its 
consideration of psychological effects, can better explain the traveler route decision-making behavior under information provision.

Table 1. Estimation results of the latent variable model

\begin{tabular}{|c|c|c|c|c|c|c|}
\hline \multirow[t]{2}{*}{ Variables } & \multicolumn{2}{|c|}{$\begin{array}{l}\text { Cognitive } \\
\text { burden }\end{array}$} & \multicolumn{2}{|c|}{$\begin{array}{l}\text { Cognitive } \\
\text { decisiveness }\end{array}$} & \multicolumn{2}{|c|}{$\begin{array}{c}\text { Emotional } \\
\text { relief }\end{array}$} \\
\hline & Est. & $t$-stat & Est. & $t$-stat & Est. & $t$-stat \\
\hline \multicolumn{7}{|l|}{ Measurement Relationship } \\
\hline The amount of information was too much to comprehend. $\left(\mathrm{Z}^{1}\right)$ & 1.000 & - & - & - & - & - \\
\hline The content of information was difficult to comprehend. $\left(Z^{2}\right)$ & 1.899 & 4.602 & - & - & - & - \\
\hline $\begin{array}{l}\text { Having information from multiple sources made it difficult to } \\
\text { comprehend the information. }\left(\mathrm{Z}^{3}\right)\end{array}$ & 0.050 & 2.424 & - & - & - & - \\
\hline $\begin{array}{l}\text { The content of information was consistent with my travel time } \\
\text { expectations up to now for the remainder of the trip. }\left(\mathrm{Z}^{4}\right)\end{array}$ & - & - & 1.000 & - & 0.038 & 4.764 \\
\hline $\begin{array}{l}\text { The content of information from multiple sources was } \\
\text { consistent. }\left(Z^{5}\right)\end{array}$ & - & - & 0.325 & 2.800 & - & - \\
\hline $\begin{array}{l}\text { The content of information was sufficient to make a decision. } \\
\left(Z^{6}\right)\end{array}$ & - & - & 6.411 & 3.835 & - & - \\
\hline $\begin{array}{l}\text { The content of information was favorable to my travel } \\
\text { situation. }\left(Z^{7}\right)\end{array}$ & - & - & - & - & 1.000 & - \\
\hline \multicolumn{7}{|l|}{ Structural Relationship } \\
\hline Female & 0.571 & 1.882 & - & - & -5.500 & -1.988 \\
\hline Age & $\mathbf{0 . 0 3 3}$ & 4.048 & - & - & 0.103 & 0.907 \\
\hline High household income & -1.510 & -3.026 & - & - & - & - \\
\hline Alternative route provided in GPS & -1.025 & -3.409 & - & - & 6.258 & 2.053 \\
\hline High amount of information & 1.119 & 2.136 & 0.988 & 3.965 & - & - \\
\hline Alternative route information & - & - & 1.101 & 6.509 & - & - \\
\hline
\end{tabular}

Estimates in bold: statistically significant at the $95 \%$ confidence level $(\mathrm{p}<0.05)$

Table 2. Estimation results of the discrete choice model

\begin{tabular}{|c|c|c|c|c|}
\hline \multirow[b]{2}{*}{ Variables } & \multicolumn{2}{|c|}{$\begin{array}{c}\text { Binary logit model } \\
\text { without latent variables }\end{array}$} & \multicolumn{2}{|c|}{$\begin{array}{l}\text { Hybrid choice model } \\
\text { with latent variables }\end{array}$} \\
\hline & Est. & $t$-stat & Est. & $t$-stat \\
\hline Constant & 0.053 & 0.147 & -0.044 & -0.145 \\
\hline \multicolumn{5}{|l|}{ Observed variables } \\
\hline Female & -0.189 & -1.102 & - & - \\
\hline Age & 0.002 & 0.235 & - & - \\
\hline High household income & -0.092 & -0.350 & - & - \\
\hline Alternative route provided in GPS & 0.207 & 1.211 & - & - \\
\hline High amount of information & -0.812 & -2.667 & - & - \\
\hline Alternative route information & 1.176 & 6.587 & - & - \\
\hline Low education & -0.426 & -1.826 & -0.447 & -1.779 \\
\hline High traffic demand & 0.896 & 5.261 & 0.926 & 4.881 \\
\hline Business trip purpose & $\mathbf{0 . 3 8 0}$ & 2.250 & 0.421 & 2.256 \\
\hline Familiarity with experiment process & -0.315 & -1.420 & -0.398 & -1.698 \\
\hline Currently using freeway & -1.521 & -7.468 & -1.624 & -7.220 \\
\hline \multicolumn{5}{|l|}{ Latent variables } \\
\hline Cognitive burden & - & - & -0.069 & -2.386 \\
\hline Cognitive decisiveness & - & - & 1.077 & 4.170 \\
\hline Emotional relief & - & - & 0.035 & 3.920 \\
\hline Number of observation & \multicolumn{2}{|c|}{722} & \multicolumn{2}{|c|}{722} \\
\hline \multicolumn{5}{|l|}{ Goodness-of-fit indexes } \\
\hline Log-likelihood & \multicolumn{2}{|c|}{-7592.575} & \multicolumn{2}{|c|}{-7077.097} \\
\hline AIC & \multicolumn{2}{|c|}{15309.150} & \multicolumn{2}{|c|}{14308.194} \\
\hline
\end{tabular}

Estimates in bold: statistically significant at the $95 \%$ confidence level $(\mathrm{p}<0.05)$ 
The roles of information perception in multiple dimensions and the psychological effects of information are identified and verified. The study results can provide system operators with insights for developing effective strategies for information creation and provision based on the holistic understanding of route choice behavior to improve system performance (such as reducing congestion).

\section{ACKNOWLEDGMENTS}

This study is based on research supported by the NEXTRANS Center, the USDOT Region 5 University Transportation Center at Purdue University. Any errors or omissions remain the sole responsibility of the authors.

\section{REFERENCES}

Avineri, E., \& Prashker, J.N. (2006) The impact of travel information on travelers' learning under uncertainty. Transportation 33 (4), pp. 393-408.

Ben-Elia, E., Erev, I., \& Shiftan, Y. (2008) The combined effect of information and experience on drivers' route choice behavior. Transportation 35 (2), pp. 165-177.

Bollen, K.A. (2002) Latent variables in psychology and the social sciences. Annual Review of Psychology 53, pp. 605-634.

Bonsall, P. (2004) Traveler behaviour: decision-making in an unpredictable world. Journal of Intelligent Transportation Systems 8, pp. 45-60.

Chorus, C.G., Molin, E.J.E., \& Wee, B. van (2006) Use and effects of advanced traveller information services (ATIS): a review of the literature. Transportation Review 26, pp. 127-149.

Gao, S., Frejinger, E., \& Ben-Akiva, M. (2011) Cognitive cost in route choice with real-time information: an exploratory analysis. Transportation Research Part A: Policy and Practice 45 (9), pp. 916-926.

Jha, M., Madanat, S., \& Peeta, S. (1998) Perception updating and day-to-day travel choice dynamics in traffic networks with information provision. Transportation Research Part C: Emerging Technologies, 6 (3), pp. 189-212.

Nakayama, S., \& Kitamura, R. (2000) Route choice model with inductive learning. Transportation Research Record: Journal of the Transportation Research Board 1725, pp. 63-70.

Peeta, S., Ramos, J.L., \& Pasupathy, R. (2000) Content of variable message signs and on-line driver behavior. Transportation Research Record: Journal of the Transportation Research Board 1725, pp. 102-108.

Peeta, S., \& Yu, J.W. (2004) Adaptability of a hybrid route choice model to incorporating driver behavior dynamics under information provision. IEEE Transactions on Systems, Man, and Cybernetics Part A: Systems and Humans 34 (2), pp. 243-256.

Peeta, S., \& Yu, J.W. (2005) A hybrid model for driver route choices incorporating en-route attributes and real-time information effects. Networks and Spatial Economics 5 (1), pp. 21-40.

Razo, M., \& Gao, S. (2013) A rank-dependent expected utility model for strategic route choice with stated preference data. Transportation Research Part C: Emerging Technologies 27, pp. 117-130.

Srinivasan, K.K., \& Mahmassani, H.S. (2000) Modeling inertia and compliance mechanisms in route choice behavior under real-time information. Transportation Research Record: Journal of the Transportation Research Board 1725, pp. 45-53.

Walker, J., \& Ben-Akiva, M. (2002) Generalized random utility model. Mathematical Social Sciences 43, pp. 303-343.

Yu, J.W., \& Peeta, S. (2011) Experimental analysis of a hybrid route choice model to capture dynamic behavioral phenomena under advanced information systems. KSCE Journal of Civil Engineering 15 (1), pp. 175-185. 